Aim of special session Main problems related to pesticide use and abuse in the world will be addressed, with a particular effort for identifying possible solutions.

Pierluigi $\mathrm{Cocco}^{3}$, Erik Jors ${ }^{4}$, Angelo Moretto ${ }^{5}$, Leslie London $^{6}$, El Yamani Mounia ${ }^{7}$, Francesca Larese Filon ${ }^{8}$, Susan Brumby $^{9}$, Claudio Colosio ${ }^{1}$, Silvia Fustinoni ${ }^{10}$, Hanifa Denny ${ }^{2}$

${ }^{1}$ Department of Health Sciences of the University of Milan and International Centre for Rural Health of the S. Paolo Hospital, Italy

${ }^{2}$ Foundation Learning and Developing Occupational Health, Leusden, The Netherlands

${ }^{3}$ Diponegoro University, Semarang, Indonesia

${ }^{4}$ Department of Medical Sciences and Public Health, University of Cagliari, Cagliari, Italy

${ }^{5}$ Department of Occupational and Environmental Health, Odense University Hospital, Odense, Denmark

${ }^{6}$ Department of Biomedical and Clinical Sciences, University of Milan, Milan, Italy

${ }^{7}$ School of Public Health and Family Medicine, University of Cape Town, Cape Town, South Africa

${ }^{8}$ Santé publique France, Direction Santé Travail, St Maurice, France

${ }^{9}$ Unit of Occupational Medicine, Dept. of Medical Sciences, University of Trieste. Trieste, Italy

${ }^{10}$ National Centre for Farmer Health, Western District Health Service, Hamilton VIC, Australia

\section{9a DEVELOPMENT OF NEW TOOLS FOR BRINGING PESTICIDE RISK ASSESSMENT IN THE SMALL SIZE ENTERPRISES AND IN THE DEVELOPING WORLD}

C Colosio. Department of Health Sciences of the University of Milan and International Centre for Rural Health of the S. Paolo Hospital, Italy

\subsection{6/oemed-2018-ICOHabstracts.1330}

The role of pesticides in the modern society has been strengthened by the need for higher yield in food production and the ongoing battle against vector borne diseases in public health. Nevertheless, the toxicity of these chemicals is not fully specific to target organisms, thus posing a potential health threat to humans. In this frame, risk assessment and management are fundamental. In the occupational settings, variability of meteorological conditions, use of different concentrations of highboy variable mixtures, and significant variations in the application times and modalities make this task very complicated, underlining the need for novel approaches for conducting 'in field' preventive activities. New developments in pesticide risk assessment should start from the fact that the amount of information collected during the process of authorisation of new active ingredients is unique, similar to that available for pharmaceutical products, and that this significant amount of information is scarcely used in the post market risk assessment activities. In this light, a possible way forward for pesticide risk assessment is represented by a better exploitation of these data, in approaches with variable levels of complexity; the simplest, is the evaluation of the adherence of the use scenario under evaluation with the one checked and approved in the authorisation process and synthesised in the good agricultural practices. Other parameters such as
Acceptable Operator Exposure Level (AOEL), acute reference dose (ArD) as well data regarding skin absorption, metabolism and relevant metabolites in animals can find use in the realisation of models adequate to estimate the dose and the risk without doing analysis, as well as to calculate provisional biological exposure indices, defining the dose supposed to be excreted in a subject exposed at the AOEL level. This can be done conducing real-life field studies to usable refine and validate the risk hypotheses generated through modelling.

\section{9b SAFE USE OF PESTICIDES AMONG TRADITIONAL FARMERS IN JAVA INDONESIA}

Hanifa M Denny*, Bina Kurniawan*, Siswi Jayanti*, Ari Suwondo*. Diponegoro University, Semarang, Indonesia

\subsection{6/oemed-2018-ICOHabstracts. 1331}

Introduction The use of pesticides in the Indonesian vegetable farming is common. The conventional and traditional methods of pesticides used are mostly dangerous nor safe. This study investigated the impact of health promotion intervention on safe use of pesticides among farmers in Sumowono Village, Semarang District, Indonesia.

Methods The researchers conducted a multi-year's approach to conduct a training on safe use of pesticides as a health promotion intervention. In 2013, the researcher approached the community health centre staff to gather their understanding on unsafe pesticides used among farmers. The researchers made a collaboration with one nurse and one midwife to be part of the field coordinator team. The actual training session was conducted in 2015.

Results The farmer's community agreed with the idea of participating in the Focus Group Discussion and training on safe use of pesticides. Selected farmers of 136 persons participated in the training. It was tailored to find a solution on un-safe use of pesticides according to participants daily practices.

Knowledge and attitude of pesticides use were improved after joining the training session. The adoption of expected behaviour on safe use of pesticides was evaluated one year after the training. A significant behaviour change was seen on drinking water after working with pesticides. In addition, the practice of taking a painkiller medicine was no longer available since the training participants gained knowledge on dehydration, pesticides intoxication, and the benefit of water intake to expedite the excretion of pesticides from the body. Conclusion Health promotion interventions involving the local health workers were effective. A three years approach in health promotion delivery was useful in strengthening to the adoption of the expected healthy behaviours.

\section{9c CANCER AND OTHER CHRONIC DEGENERATIVE DISEASES FOLLOWING LONG TERM EXPOSURE TO PESTICIDES}

Pierluigi Cocco. Department of Medical Sciences and Public Health, University of Cagliari, Cagliari, Italy

10.1136/oemed-2018-ICOHabstracts. 1332 
Introduction The definition of pesticide covers about 100000 different chemicals, inorganic and organic, that are grouped according to their function or their chemical structure. Evidence is growing about long-term health effects following high-level, long-lasting exposure to some pesticides, including asthma and other allergic diseases, immunotoxicity, endocrine disruption, cancer, and central and peripheral nervous system effects. Identifying such specific chemicals is of paramount importance in preventing ill health in farmers, while preserving the benefits of plant protection against pests.

Methods An extensive review of the literature was conducted. Result Currently, the International Agency for Research on Cancer classifies three obsolete agrochemicals as Group 1 human carcinogens (Arsenic compounds, lindane, and 1,2dichloropropane); six are classified as probable human carcinogens, including captafol, DDT, diazinon, glyphosate, ethylene dibromide, and malathion, generating a vast controversy among the International Health and Safety Agencies and the media about the need to phase them out as some were widely used worldwide. Possibly human carcinogen pesticides are 20, and for 32 there is inadequate scientific evidence to classify their potential human carcinogenicity. The endocrine disrupting effects of numerous pesticides have also been extensively studied, but evidence of hormonal effects comes mainly from animal studies, either laboratory or wildlife animals; such results are difficult to refer to the low level long term exposure in humans. Other chronic effects repeatedly reported among users of specific pesticides include asthma and chronic bronchitis, immunosuppression, and neurodegenerative diseases, with the classical example of paraquat, but also other pesticide classes, and Parkinson's disease.

Discussion Major reasons for uncertainty in interpreting epidemiological findings of long term pesticide effects include the complex pattern of overlapping exposure due to multiple treatments applied to different crops and their frequent changes over time to overcome pest resistance. Further research will have to address specific agrochemicals with wellcharacterised exposure patterns.

\section{9d INDISCRIMINATE USE OF PESTICIDES IN AGRICULTURE AND EFFECTS ON HEALTH AND ENVIRONMENT EXAMPLES FROM BOLIVIA, UGANDA, NEPAL AND CAMBODIA!}

E Jørs. Department of Occupational and Environmental Health, Odense University Hospital, Denmark

\subsection{6/oemed-2018-ICOHabstracts. 1333}

Introduction Pesticide use is increasing in developing countries trying to boost their agricultural production. Due to lack of proper knowledge among small-holder farmers this leads to frequent poisonings of farmers and pollution of the environment. Integrated Pest Management (IPM) has been tried out to minimise the use of pesticides and a banning of the most toxic pesticides is seen in some countries.

Methods Small-holder farmers from Bolivia, Nepal, Uganda and Cambodia were interviewed in cross-sectional baseline studies before an intervention teaching farmers IPM took place. Follow-up studies were conducted after training was completed to evaluate the effect of the trainings. Sound statistical methods were applied while controlling for relevant confounders. Ethical approval was obtained.
Results At baseline farmers used generally very toxic pesticides, most pronounced in Bolivia. Farmers had little knowledge and use of personal protection when handling pesticides, and use of alternatives to pesticides were scarce. Pesticide poisonings were commonly reported and an effect with a lowered blood level of AChE was seen depending on spraying frequency, use of PPE and the toxicity of the pesticides used. Training improved farmer's knowledge and practice on pesticide handling and IPM and lowered the number of symptoms of poisoning after spraying. Obstacles for a wider diffusion of IPM seem to be an extra workload not always compensated for by higher prices of the products.

Conclusion Pesticide poisonings is increasing among farmers from low income countries, leading to acute symptoms eventually dead or chronic poisonings with neurological impairment, cancer, lung diseases or skin diseases and fetal damage. Pesticide abuse can be prevented without the loos of crops by teaching farmers integrated pest management methods and political interventions with a ban of the most dangerous pesticides nationally and worldwide have proved effective as well.

\section{9e PESTICIDE PREVENTION STARTS FROM THE PRE- MARKETING PHASE}

A Moretto. Department of Biomedical and Clinical Sciences, University of Milan, Italy

\subsection{6/oemed-2018-ICOHabstracts.1334}

Pesticide active ingredients are extensively studied before being authorised for use. These include, among others, efficacy studies, several toxicity studies in mammals and in ecotoxicological relevant species. From these studies, health based limits for the consumer are derived such as the acceptable daily intake (ADI), the acute reference dose (ARfD) and the exposure limits for pesticide operators (AOEL); also, environmental limits are defined and levels of residues in food commodities determined.

Then the exposure for consumer and operators is estimated by using different mathematical models based on the proposed uses scenario (e.g: application doses, period and interval between application, etc). A further step is to compare the estimated exposure with the reference value to assess the risk and conclude if the proposed use is safe, therefore the active ingredient can be put on the market, or not. It has to be underlined that the use scenario for exposure estimation is always based on a worst-case approach to cover all possible conditions.

In particular, estimation of operators' exposure is made on mathematical models based on dermal and inhalation exposures measured following different type of application in several field studies.

Finally, if safe uses have been identified, approval of an active ingredient and of a commercial formulation is consequently accompanied by indication of the proper application modalities and PPE to be used by the pesticide applicator to reflect the scenario evaluated.

Moreover, the risk assessment is conducted not only for consumer and operators but also for workers re-entering the treated crops, bystander, and especially resident.

Therefore, it can be concluded that if an active ingredient is used on crops according the Good Agricultural Practice and according all indications on the label (e.g.: use of specific PPE, buffer zone, post harvesting interval), negligible risk for human and environment is expected. 